\title{
Aplicação da Bioética na prática clínica diária
}

Application of Bioethics in daily clinical practice

Janine Julieta Inocente

Doutora em Psicologia

Aluna de Especialização em Saúde Coletiva e da Família da UFRJ

\section{Urubatan Medeiros}

Doutor pela USP

Professor Titular do Departamento de Odontologia Preventiva e Comunitária da UERJ/UFRJ

\section{Resumo}

A Bioética é a parte da ética, ramo da filosofia, que enfoca os limites e as finalidades de intervenção do ser humano sobre a vida, identifica os valores de referência racionalmente proponiveis e denuncia os riscos das possiveis aplicações. 0 presente estudo teve por objetivo refletir sobre a bioética na prática odontológica e para tal foi realizada uma busca não exaustiva na literatura e uma análise dos estudos sobre a bioética na prática odontológica, delineando pontos a serem explorados dentro da Odontologia. Conclui-se, com base nos estudos utilizados, que a sociedade contemporânea necessita mais do que nunca de cidadãos éticos e que os cirurgiões-dentistas viabilizem um trabalho mais humanizado, viável, baseado nos princípios éticos.

Palavras-chave: Bioética; Bioética em Odontologia.

\section{Abstract}

Bioethics is the part of ethics, philosophy branch, which focuses on the limits and purposes of human intervention on life, identifies rationally reference values and denounces the risks of possible applications. This study aimed to reflect on bioethics in dental practice. A literature review and an analysis of studies on bioethics in dental practice was conducted. We conclude, based on the literature, that contemporary society needs more than ever of ethical citizens and theDentists enable a more humanized work, viable, based on ethical principles. Keywords: Bioethics; Bioethics in Dentistry.

\section{Introdução}

$s$ avanços científicos e tecnológicos no campo da biomedicina exigiram uma reflexão ética e é neste contexto que a bioética se apresenta como estudo e reflexão ético-moral (1).

Em 1970, apareceu o vocábulo "bioética" no artigo escrito por Van Rensselaer Potter, da Universidade de Wisconsin, investigador da bioquímica do câncer, preocupado com o progresso da ciência, da sociedade e a capacidade de sobrevivência da humanidade $(2,3)$. A Bioética é a ética aplicada à vida, campo de ação e de interação de profissionais e estudiosos de diversas áreas do conhecimento humano (4).

A bioética é a parte da Ética, ramo da filosofia, que enfoca as questões sobre saúde (4). Ela é compreendida como um movimento cultural que engloba problemas referentes aos direitos e obrigações dos profissionais, dos pacientes e da sociedade, relacionados à saúde (5).

A bioética realiza a "análise racional dos problemas morais ligados à biomedicina e de sua conexão com as áreas do direito e das ciências humanas" (2). Ainda, consiste em um estudo sistemático da moralidade das tecnociências da vida e da saúde, com ênfase nos valores e princípios morais (6).

PUPLAKSIS et al. (7) enfatizam que o ensino de bioética desenvolve princípios ligados ao crescimento da tecnologia, da investigação científica, da resolução de conflitos morais e de transformar a sociedade em mais justa.

A bioética busca a humanização dos serviços de saúde e promoção dos direitos dos usuários (8). É uma disciplina transdisciplinar que propõe a análise e mediação dos conflitos gerados nas áreas de conhecimento relacionadas às ciências biomédicas e da saúde (9).

Dessa maneira, os processos saúde-doença bucal podem gerar conflitos na prática odontológica quanto: ao paciente, que na sua individualidade, deve atuar dentro de uma sociedade democrática, guiado pelos princípios da autonomia e do direito; quanto à comunidade, diz respeito ao coletivo e que deve funcionar a partir do princípio da justiça; quanto ao cirurgião-dentista, refere-se à categoria profissional na qual faz parte e que deve atuar pelos referenciais da beneficência, da não maleficência e da qualidade; e, por último, quanto ao Estado, pelo dever ou responsabilidade (5).

Além do conhecimento teórico e da habilidade clínica, o cirurgião-dentista precisa estar bem informado sobre outros aspectos importantes do exercício da profissão. Um deles é a responsabilidade civil na Odontologia, que diz respeito às obrigações de ordem civil, penal, ética e administrativa, às quais o profissional está sujeito na relação com seus pacientes (10).

Os aspectos bioéticos, éticos e legais não devem ser negligenciados pelos cirurgiões-dentistas durante o tratamento odontológico, pois este não se restringe apenas ao ato clínico, mas abrange desde a relação profissional/paciente até a documentação odontológica. Além disso, todos os cidadãos têm o direito de questionar uns aos outros judicialmente, caso acreditem que tenham sofrido um dando. Este é um dos pilares do Direito Civil. Uma vez que é impossível evitar um processo caso o paciente assim o deseje, é importante que o profissional esteja bem documentado para comprovar a conduta adequa- 
da, especialmente nas situações em que seja essencial que o paciente dê o seu consentimento. Todas as especialidades estão sujeitas à questionamentos judiciais, mas as áreas de Implantodontia, Ortodontia, Prótese Dental, Cirurgia Bucomaxilofacial e Endodontia são as especialidades mais envolvidas nos processos cíveis, ou seja, naqueles em que o paciente busca por uma indenização ou reparação de danos (10).

De acordo com COELHO-FERRAZ et al. (11), o conhecimento da vida e o conhecimento dos valores norteiam o nosso agir e torna-se essencial e persistente para uma prática mais humana tendo sempre em mente que qualquer exercício das relações profissionais em saúde tornam-se destinatários do discurso bioético.

O Código de Ética Odontológica (CEO) brasileiro aprovado pela Resolução CFO-118/2012 tem no seu capítulo I, Art. $2^{\circ}$ : A Odontologia é uma profissão que se exerce em benefício da saúde do ser humano, da coletividade e do meio ambiente, sem discriminação de qualquer forma ou pretexto. No seu Art. $3^{\circ}$ enfatiza que: O objetivo de toda a atenção odontológica é a saúde do ser humano. Caberá aos profissionais da Odontologia, como integrantes da equipe de saúde, dirigir ações que visem satisfazer as necessidades de saúde da população e da defesa dos princípios das políticas públicas de saúde e ambientais, que garantam a universalidade de acesso aos serviços de saúde, integralidade da assistência à saúde, preservação da autonomia dos indivíduos, participação da comunidade, hierarquização e descentralização político-administrativa dos serviços de saúde.

PYRRHO et al. (9) analisaram o Código de Ética Odontológica (CEO) brasileiro comparando os enfoques deontológico e bioético em doze textos selecionados, seis sobre bioética e seis sobre deontologia, quanto ao principialismo bioético, a saber: autonomia, beneficência, não maleficência e justiça, considerando os aspectos técnicos e virtudes morais relacionados à profissão. Os resultados indicaram que os quatro princípios somados representaram 22,9\%, 39,8\% e $54,2 \%$ do conteúdo do CEO, dos textos deontológicos e dos bioéticos, respectivamente. No CEO, $42 \%$ dos itens referiam-se às virtudes, $40,2 \%$, a aspectos técnicos e $22,9 \%$, aos princípios. As virtudes relacionadas aos profissionais e os aspectos técnicos juntos representaram $70,1 \%$ do código. Os autores concluíram que o CEO, em vez de centrar-se no paciente como sujeito do processo de atenção à saúde bucal, focaliza o profissional, sendo voltado para os aspectos legalistas e corporativistas.

A Bioética é a ética prática que analisa casos, interpreta técnicas e programas, confronta ideias e argumenta com base na razão. A ética em saúde bucal é compreendida em duas vertentes: macro e micro. Na vertente macro, consiste em um conjunto de decisões e medidas técnicas, políticas e sanitárias que proporcionam ampliação da cidadania e di- minuição da exclusão social; na vertente micro, é o resultado moral do conjunto de ações clínicas, individuais e coletivas, visando melhorar a qualidade de vida e níveis de bem-estar e felicidade (5).

\section{Resultados e Discussão}

A seguir serão apresentados estudos relacionados à bioética na Odontologia desde a formação do aluno até a prática clínica.

\section{Formação Odontológica}

No que se refere ao ensino da Bioética no curso de Odontologia, MUSSE et al. (12) verificaram sobre o oferecimento da disciplina nos cursos de graduação em Odontologia do Estado de São Paulo, por meio da observação de suas respectivas estruturas curriculares. Os resultados obtidos no estudo evidenciaram lacunas quanto ao ensino da Bioética, pois dos 47 cursos pesquisados, apenas quatro possuem a disciplina em sua grade curricular. Nestas, a disciplina é ministrada por docentes com inserção na área de Bioética. Observaram que em três delas, o oferecimento da disciplina ocorre a partir da metade do curso e em uma no primeiro ano. Os autores concluíram que há a necessidade de se aumentar a carga horária e de se criar a disciplina de Bioética nos cursos de graduação em Odontologia para que o aluno possa refletir e tomar decisões, pautado em princípios éticos.

PUPLAKSIS et al. (7) realizaram duas pesquisas na Faculdade de Odontologia da Universidade de São Paulo (FOUSP), para verificar a contribuição da disciplina Bioética para a formação humanística dos alunos: Estudo 1, em 2004, trabalhou com os alunos que não tinham aulas sobre Bioética e Estudo 2, em 2007, trabalhou com os alunos matriculados na disciplina, indagando sobre a qualidade da relação terapêutica e a relação profissional/paciente dos serviços odontológicos oferecidos pelos alunos. Os resultados mostram que a inclusão da Bioética como disciplina obrigatória do currículo é um passo importante na mudança sobre o valor da dignidade humana. Os autores concluíram que a Bioética não deve ser somente uma disciplina isolada da transmissão de valores e que os professores ensinem a aplicação de valores éticos na relação profissional/paciente. O contexto educacional é essencial para a educação integral humanística do aluno.

No estudo realizado por MACPHERSON et al. (13), os professores relataram dificuldades e tentativas frustrantes para concretizar a intenção de formar profissionais com valores éticos. Para eles, muitos alunos são refratários a essas mensagens, de modo que os valores construídos ao longo de suas vidas, destacadamente no âmbito familiar, são importantes determinantes nas atitudes assumidas. Assim, eles revelam as suas limitações em contribuir com a formação de alguns alunos em sua dimensão ética.

De acordo com FINKLER et al. (11), ocorreu um comprometimento pontual dos cursos com a dimensão ética 
da formação profissional, bem como avanços em relação à capacitação docente, ao perfil do egresso e ao processo de integração curricular. Por outro lado, há deficiências quanto aos conteúdos relacionados à formação cultural, humanística e política, em relação à orientação didática e aos cenários de ensino-aprendizagem, ao processo avaliativo, além da incipiente presença da Bioética enquanto disciplina e tema transversal curricular, acompanhada pela escassa formação específica de seus docentes.

Em outro estudo realizado pelos mesmos autores, o embasamento no senso comum e a ausência de intencionalidade do corpo docente com relação à formação ética dos estudantes indicam como imperativa a necessidade de se conhecer os valores que vivenciam, de se entender como ocorre o desenvolvimento moral e de se aproximar de um referencial bioético para fundamentar e instrumentalizar o fazer ético-pedagógico (15).

Um estudo realizado por JANAKIRAM et al. (16) avaliou o conhecimento de 209 estudantes de Medicina e Odontologia em relação às práticas e atitudes com a ética. Quase 68\% dos pós-graduados não tinham sofrido quaisquer problemas com a bioética. Quase $98 \%$ dos pós-graduados médicos, em comparação com $79 \%$ dos pós-graduados em Odontologia, sabiam que a sua instituição tem um comitê de ética. Houve diferença entre os estudantes de Medicina e de Odontologia em termos de suas atitudes e conhecimentos da ética e de cuidados de saúde, com o primeiro tendo um conhecimento superior sobre o assunto e uma atitude melhor. Os pós-graduados médicos e odontológicos se deparam com questões éticas durante sua formação, mas não estão preparados para resolverem os dilemas éticos que encontram.

Em um estudo atual (17) foi identificado em 14 estudantes de Odontologia as representações de valores morais considerados centrais para o exercício profissional. Os resultados indicaram que, para a maioria deles, a ética se relaciona com normas, condutas e princípios impostos pela sociedade a todos os indivíduos, enquanto a moral, por ser mais subjetiva, diz respeito à forma de pensar e agir do ser humano no plano individual. Ainda relataram respeito, honestidade e humildade como os principais valores morais para o exercício profissional. Os autores concluíram que é importante repensar a ética pedagógica adotada no processo de formação acadêmica, visando alternativas de trabalho humanizadoras, capazes de vivenciar e de promover a realização dos valores humanos.

\section{Observação na Clínica Odontológica}

GONÇALVES \& VERDI (18) advertem que, com o advento do HIV/AIDS, o comércio de dentes humanos, as pesquisas que descobrem novas técnicas e biomateriais, os estudos com células troncos, os paradoxos entre políticas públicas de saúde e justiça social e a constante busca da humanização e do respeito aos princípios éticos na relação entre profissional e paciente são exemplos de conflitos éticos na Odontologia. Com isso, a formação de futuros profissio- nais é a importante missão das instituições de ensino.

Um estudo identificou, a partir da visão dos cirurgiões-dentistas, os problemas éticos vivenciados na prática odontológica. O material empírico foi coletado através de entrevistas semiestruturadas, realizadas com quinze cirurgiões-dentistas. Os resultados apontaram que muitos dos problemas éticos coincidem com infrações ao Código de Ética Odontológica, confirmando uma noção de ética deontológica adquirida na formação profissional e, portanto, insuficiente para solucionar os problemas que emergem na prática profissional. Os problemas éticos identificados na prática profissional precisam ser compreendidos para além da dimensão deontológica em direção aos aspectos da produção do trabalho. Torna-se preciso, então, incorporar nas práticas de saúde, incluindo as de saúde bucal, as tecnologias da gestão do cuidado, o que implica o reconhecimento de diferentes dimensões que produzem os sujeitos e suas necessidades de saúde (8).

A noção de ética deontológica, adquirida na formação profissional, não é suficiente para solucionar os problemas que emergem na prática profissional. É preciso uma maior preocupação em preservar a autonomia dos usuários, a garantia do acesso aos serviços de referência especializada, a necessidade de realizar somente procedimentos para os quais estiverem capacitados tecnicamente (19).

Em determinadas áreas da Odontologia, especialmente nas que envolvem as partes estética e reabilitadora, existe um grande desnível entre a expectativa do paciente para o resultado final e o real prognóstico do caso. O CD deve sempre buscar o equilíbrio entre estes dois pontos. Outra situação que gera problemas jurídicos é quando os cirurgiões-dentistas permitem que seus pacientes sejam atendidos por técnicos sem a devida supervisão (10).

A atualização do prontuário odontológico é uma conduta simples que costuma ser negligenciada. Todas as etapas do tratamento odontológico devem ser documentadas. Os exames de imagem também têm relevância para evitar casos de responsabilidade civil se forem bem documentados e anexados aos prontuários dos pacientes. Além disso, a documentação digital é bem aceita como meio de prova, inclusive as fotografias digitais (10).

No que se refere ao sigilo odontológico, ele só pode ser quebrado mediante autorização do usuário ou nas situações amparadas legalmente, consideradas como justa causa. É preciso discutir o tema com os trabalhadores de saúde (20).

Foi realizada uma análise comparativa dos códigos de ética de três organizações: Código de Ética para Dentistas na União Europeia, elaborado pelo Conselho de Dentistas Europeus (CED); Código de Ética Español y Deontología Dental, publicado pelo Consejo General de Colegios de Odontologos y Estomatólogos de España (CGCOE); e Princípios de Ética e Código de Conduta Profissional, da American Dental Association (ADA). A análise da estrutura dos códigos permite a descoberta de diferentes abordagens que regem a ética profissional de acordo com a tradição ética e 
legislativa a partir do qual elas derivam. Enquanto existem elementos comuns inerentes à cultura ocidental, há nuances nos fundamentos expostos e que refletem problemas reais enfrentados pelos cirurgiões-dentistas no exercício de sua profissão (13).

GONÇALVES (21) avaliou o conhecimento de 163 cirurgiões-dentistas. Em relação aos aspectos bioéticos, 88,1\% citam que a decisão sobre o tratamento deve ser resultado em comum acordo entre o profissional e o paciente, porém $26,4 \%$ relatam que a participaçãodo paciente e/ou seu representante legal pode interferir de maneira negativa quando os mesmos optam pelo tratamento menos vantajoso. Já a atuação do profissional, quando o paciente escolhe um tratamento menos conveniente, $95,6 \%$ dos entrevistados tenta convencê-lo a optar pelo tratamento melhor. Os principais documentos mencionados pelos entrevistados e que compõem o prontuário dos mesmos foram: anamnese (78,9\%), odontograma (61,7\%), plano de tratamento $(58,6 \%)$, identificação $(42,1 \%)$ e radiografias $(32,3 \%)$.

Em relação ao uso de biomateriais, foram avaliados os aspectos relacionados aos critérios de utilização, riscos, origem, comercialização, controle sanitário e participação do paciente na escolha terapêutica através de 95 cirurgiões-dentistas. Os resultados obtidos demonstraram que os entrevistados utilizavam rotineiramente biomateriais, sendo que $45 \%$ acreditam não haver riscos para os pacientes; $48 \%$ não consideram o biomaterial como medicamento e $70 \%$ se sentiam seguros com relação à origem, apesar de membranas e osso serem os itens mais adquiridos por meio de vendedores autônomos. Apesar disso, 96\% dos entrevistados afirmaram que deveria haver maior controle das autoridades sanitárias. Mais da metade dos entrevistados (51\%) referiu pouca ou nenhuma participação do paciente no processo de escolha terapêutica. Os autores concluíram que os profissionais utilizam biomateriais desconhecendo riscos e efeitos adversos, distanciando-se do princípio da beneficência. Ainda relataram que a ética da responsabilidade pública não é observada pelos organismos públicos responsáveis e órgãos de categoria profissional. Advertiram que o consentimento informado ainda não está incorporado à prática profissional, permanecendo a relação profissional-paciente em Odontologia excessivamente vertical (1).

\section{Conclusão}

Conclui-se, com base na literatura consultada, que a sociedade contemporânea necessita mais do que nunca de cidadãos éticos e que os cirurgiões-dentistas viabilizem um trabalho mais humanizado, viável, baseado nos princípios éticos.

Sugere-se a incorporação de um currículo de bioética desde o primeiro ano do curso de graduação, além dos programas de pós-graduação, para reforçar os procedimentos éticos na prática odontológica.

Além disso, torna-se fundamental a implementação de debates nesta área para que possam produzir reflexões que ajudem os profissionais da Odontologia a conduzirem essa dimensão sólida na pratica odontológica. 
Referências ::

1. Bugarin Júnior Jg, Garrafa V. Bioética e biossegurança: uso de biomateriais na prática odontológica. Rev Saúde Pública. 2007; 41(2).

2. Sgreccia E. Manual de bioética. I. Fundamentos e ética médica. 2. ed. São Paulo: Edições Loyola, 2002.

3. Galindo GC. Bioética para odontólogos. Univ Odontol. 2005; 25(6):41-44.

4. Segre M. Definição de bioética e sua relação com a Ética, Deontologia e Diseologia. In: Sgre M, Cohen C. (Orgs.). Bioética. 3. ed. Editora da Universidade de São Paulo, 2002.

5. Garrafa V. A dimensão ética de saúde pública. São Paulo: Faculdade de Saúde Garrafa, V. Bioética e Odontologia. In: KRIGER, L. Promoção de Saúde Bucal. São Paulo: Artes Médicas, 1997.

6. Schramm FR. As diferentes abordagens da Bioética. In: Palácios M, Martins A, Pegoraro O. (Orgs.). Ética, Ciência e Saúde: desafios da bioética. Petrópolis, RJ: 2001.

7. Puplaksis NV, Pereira MM, Nobile R, et al. A disciplina de bioética na Faculdade de Odontologia da Universidade de São Paulo. Revista Latinoamericana de Bioética. 2010; 10(2).

8. Amorim AG, Souza ECF. Problemas éticos vivenciados por dentistas: dialogando com a bioética para ampliar o olhar sobre o cotidiano da prática profissional. Ciênc. Saúde Coletiva. 2010; 15(3).

9. Pyrrho M, Prado MM, Jorge Cordón J, et al. Análise bioética do Código de Ética Odontológica brasileiro. Ciência \& Saúde Coletiva. 2009; 14(5):1911-8.

10. Jornal da ABO. Ano XXII, edição 153, mai/jun/jul 2015. S-Ca

11. Coelho-Ferraz MJP, Valvassori A, Queluz DP, et al. Paradigmas da Bioética no cuidado à saúde bucal. Centro Universitário São Camilo. 2009; 3(1):117-20.

12. Musse JO, Boing AF, Martino FS, et al. O Ensino da bioética nos cursos de graduação em odontologia do estado de São Paulo. Arq Ciênc Saúde. 2007; 14(1):13-6.
13. Macpherson Mayol I, Roqué Sanchez MV, Gonzalvo-Cirac M, et al. Comparative study of three Western models of deontological codes for dentists. Cuad Bioet. 2013; 24(82):367-76.

14. Finkler M, Caetano JC, Ramos FRS. A dimensão ética da formação profissional em saúde: estudo de caso com cursos de graduação em odontologia. Ciênc. Saúde Coletiva. 2011; 16(11).

15. Finkler M, Caetano JC, Ramos FRS. Ética e valores na formação profissional em saúde: um estudo de caso. Ciênc. Saúde Coletiva. 2013; 18(10).

16. Janakiram C, Gardens SJ. Knowledge, attitudes and practices related to healthcare ethics among medical and dental postgraduate in South India. Indian J Med Ethics. 2014; 11(2):99-104.

17. Orestes-Cardoso S, Melo MVS, Orestes-Carneiro R. Representação de valores morais para o exercício profissional em estudantes de odontologia. Rev Bioét. 2015; 23(1):178-86.

18. Gonçalves ER, Verdi MIM. Os problemas éticos no atendimento a pacientes na clínica odontológica de ensino. Cienc Saúde Col. 2007; 12(3):755-64

19. Amorim AG. Bioética e Odontologia: um perfil dos problemas éticos vividos por cirurgiões-dentistas. Dissertação de Mestrado em Programa de Pós-Graduação de Odontologia Preventiva e Social, da Universidade Federal do Rio Grande do Norte. Natal, 2005.

20. Dias OV. Segredo Professional e sua importância na pratica de enfermeiros e odontológicos. Rev Bioética. 2013; 21(3):448-54.

21. Gonçalves PE. Avaliação do conhecimento dos cirurgiões-dentistas que realizam curso de especialização na Universidade Estadual Paulista- UNEP, sobre aspectos bioéticos, éticos e legais no tratamento odontológico. Dissertação apresentada ao Programa de Pós-Graduação em Odontologia Preventiva e Social da Faculdade de Odontologia de Araçatuba, Universidade Estadual Paulista, como parte dos requisitos para obtenção do título de Mestre, 2005.

Recebido em: 07/08/2015 / Aprovado em: 11/09/2015

Janine Julieta Inocente

Rua Tirol, n 450, apartamento 1302, Freguesia- Jacarepaguá

Rio de Janeiro/RJ - Brasil, CEP: 22750-009

E-mail: janineinocente@hotmail.com 